\title{
Numerical Simulations of Space-Time Conditional Random Fields of Ground Motions
}

\author{
Robert Jankowski \\ Faculty of Civil and Environmental Engineering, Gdańsk University of Technology, \\ ul. Narutowicza 11/12, 80-952 Gdańsk, Poland \\ jankowr@pg.gda.pl
}

\begin{abstract}
The aim of the present paper is to propose a method of conditional stochastic simulation of propagation of seismic wave using the spatiotemporal correlation function. The method has been used to generate unknown time histories at various points of ground motion random filed based on the specified earthquake record at one location. The results of the study show that the method considered gives relatively low simulation errors.
\end{abstract}

\section{Introduction}

Simulation of spatiotemporal variation of earthquake field by the deterministic approach is very complex. It requires detailed knowledge of the fault size, rupture mechanisms, propagation paths, distance from the epicentre, local geological and topographical conditions. Therefore in earthquake engineering, the stochastic approach has been proved to be very useful and successfully applied in practice [4].

The incorporation of the seismic wave propagation effect is especially important when large structures such as long bridges, dams, life-line systems and large buildings, are analyzed (see [3], for example). This is due to the fact that their dynamic response can be significantly altered due to the spatial variations of ground motion causing different seismic input acting on different supports of the structure. Detailed analysis of the response of large structures often requires the generation of acceleration records for different locations of supports based on the specified record. This can be achieved by applying the conditional stochastic modelling approach (see [1], [2]).

One of the problems of methods for random field simulation of ground motions concerns the issue of identifying the time correlation of the field. This problem is often simplified by the use of time-delay parameters, which are applied to shift earthquake records in time for different locations (see [2], [3]). It requires the specification of direction of the seismic wave propagation, what is reasonable in the case of line structures but may be problematic in other cases. Moreover, the simulation results obtained in this way might depend on a value of the time step used in the analysis [2].

The aim of the present paper is to propose a method of conditional stochastic simulation of ground motions using the spatiotemporal correlation function. The method has been used to generate unknown acceleration time histories at various field points based on the specified earthquake record at one location. 


\section{Conditional Random Field Simulation Method}

Let us define a correlation in space and time of the ground motion field by a spatiotemporal correlation function of the form (compare with [2]),

$$
K\left(\mathbf{r}_{i j}, t_{i j}\right)=\sigma^{2} \exp \left(-\frac{\omega_{d}\left|\mathbf{r}_{i j}\right|}{2 \pi v d}\right) \exp \left(-\beta \Delta t_{i j}\right)
$$

where $\sigma$ is a standard deviation of the earthquake record, $\omega_{d}$ is a predominant frequency of ground motion, $\left|\mathbf{r}_{i j}\right|$ is a distance between the field points $i, j, v$ is a mean apparent seismic wave velocity in the field, $d$ is a space scale parameter $(d>0)$ depending on local ground conditions, $\Delta t_{i j}$ is a time lag between the values in the ground motion records for the field points $i, j$ and $\beta$ is a time scale parameter $(\beta>0)$ describing the degree of time correlation of the field. The scale parameters $d$ and $\beta$ should be obtained experimentally based on the data concerning the seismic wave propagation patterns in the ground. It should be mentioned, that defining the correlation of the ground motion field by the use of the spatiotemporal correlation function of Eq. (1) does not involve the necessity of specifying the direction of the seismic wave propagation and of selecting the appropriate value of the time step in the earthquake record.

In the random fields theory, the earthquake acceleration record is usually treated as a set of measured values, which satisfy zero-mean Gaussian distribution. A formula for the probability density of Gaussian conditional distribution can be written as [1],

$$
f(\mathbf{x} \mid \mathbf{y})=\left(\operatorname{det} \mathbf{K}_{c}\right)^{-\frac{1}{2}} \cdot(2 \pi)^{-\frac{n}{2}} \cdot \exp \left(-\frac{1}{2}\left(\mathbf{x}-\mathbf{m}_{c}\right)^{T} \mathbf{K}_{c}^{-1}\left(\mathbf{x}-\mathbf{m}_{c}\right)\right),
$$

where $\mathbf{x}=\left[x_{1}, x_{2}, \cdots, x_{n}\right]^{T}$ is a $n$-dimensional vector of unknown values, $\mathbf{y}=\left[y_{1}, y_{2}, \cdots, y_{n^{\prime}}\right]^{T}$ is a $n^{\prime}$-dimensional vector of known values, $\mathbf{K}_{c}$ is a conditional covariance matrix,

$$
\mathbf{K}_{c}=\mathbf{K}_{11}-\mathbf{K}_{12} \mathbf{K}_{22}^{-1} \mathbf{K}_{21},
$$

and $\mathbf{m}_{c}$ is a vector of conditional mean values,

$$
\mathbf{m}_{c}=\mathbf{K}_{12} \mathbf{K}_{22}^{-1} \mathbf{y},
$$

where $\mathbf{K}_{11}, \mathbf{K}_{12}, \mathbf{K}_{21}, \mathbf{K}_{22}$, are the elements of the covariance matrix of the field determined for all (known and unknown) values,

$$
\mathbf{K}=\left[\begin{array}{ll}
\mathbf{K}_{11} \mathbf{K}_{12} \\
\mathbf{K}_{21} \mathbf{K}_{22}
\end{array}\right]_{\left(n+n^{\prime}\right) \times\left(n+n^{\prime}\right)} .
$$


The following numerical procedure, based on the acceptance-rejection theorem (compare with [1], [2]), has been used in this paper for the simulation purposes:

1. Determination of the covariance matrix of the field, $\mathbf{K}$, according to Eq.(5) assuming the spatiotemporal correlation function defined by Eq.(1).

2. Calculation of the conditional covariance matrix, $\mathbf{K}_{c}$, from Eq.(3).

Points 3-6 are repeated for all time steps in the earthquake record:

3. Generation of an unknown vector $\mathbf{x}, x_{i}=-a_{\max }+2 a_{\max } \cdot r_{d}$, where $a_{\max }$ is a peak ground acceleration and $r_{d}$ is a random variable from interval $\langle 0,1\rangle$.

4. Calculation of vector of conditional mean values, $\mathbf{m}_{c}$, according to Eq.(4), and value of conditional density function, $f(\mathbf{x} \mid \mathbf{y})$, defined by Eq.(2).

5. Generation of a random value, $R$,

$$
R=\left(\operatorname{det} \mathbf{K}_{c}\right)^{-\frac{1}{2}} \cdot(2 \pi)^{-\frac{n}{2}} \cdot r_{d} .
$$

6. Verification of the condition (von Neumann elimination),

$$
R \leq f(\mathbf{x} \mid \mathbf{y}) .
$$

If this condition holds, vector $\mathbf{x}$ is accepted and the simulation for the next time step is undertaken. If not, the calculation returns to point 3 .

7. Determination of the mean generated vector, $\overline{\mathbf{x}}$, mean known vector, $\overline{\mathbf{y}}$, and the generated covariance matrix, $\overline{\mathbf{K}}$,

$$
\overline{\mathbf{x}}=\frac{1}{N R} \sum_{i=1}^{N R} \mathbf{x}_{i}, \overline{\mathbf{y}}=\frac{1}{N R} \sum_{i=1}^{N R} \mathbf{y}_{i}, \overline{\mathbf{K}}=\frac{1}{N R-1} \sum_{i=1}^{N R}\left(\left[\begin{array}{l}
\mathbf{x}_{i} \\
\mathbf{y}_{i}
\end{array}\right]-\left[\begin{array}{l}
\overline{\mathbf{x}} \\
\overline{\mathbf{y}}
\end{array}\right]\right)\left(\left[\begin{array}{l}
\mathbf{x}_{i} \\
\mathbf{y}_{i}
\end{array}\right]-\left[\begin{array}{l}
\overline{\mathbf{x}} \\
\overline{\mathbf{y}}
\end{array}\right]\right)^{T},
$$

where $N R$ is a number of time steps in the earthquake record.

8. Comparison between the assumed covariance matrix, $\mathbf{K}$, and the generated one, $\overline{\mathbf{K}}$, by calculation the global error,

$$
G E=\left|\frac{\|\mathbf{K}\|-\|\overline{\mathbf{K}}\|}{\|\mathbf{K}\|}\right| \cdot 100 \%
$$

where $\|\mathbf{K}\|$ and $\|\overline{\mathbf{K}}\|$ are the Euclidean norms for $\mathbf{K}$ and $\overline{\mathbf{K}}$, respectively.

\section{Numerical Example}

In this paper, the example of numerical simulations of ground motion records at the corners of a $100 \mathrm{~m}$ long and $50 \mathrm{~m}$ wide building has been presented. The method has been used to generate time histories at 3 corners described by the coordinates: $(0,50)$, $(100,50)$ and $(100,0)$ based on a specified earthquake record at $(0,0)$. In the analysis, the generation of values at every time step is based on the known value at this time 
step for a specified time history as well as the known values (previously generated) for a previous time step for all records. For the simulation purposes, the N-S component of the El Centro earthquake (May 18, 1940), with the time step of $\Delta t=0.01 \mathrm{~s}$, has been used. The examples of the results of simulations obtained for $v=1000 \mathrm{~m} / \mathrm{s}$, $d=1$ and $\beta=1$ are shown in Fig. 1. The mean simulation error calculated according to Eq. (9) has reached the value of $1.78 \%$.
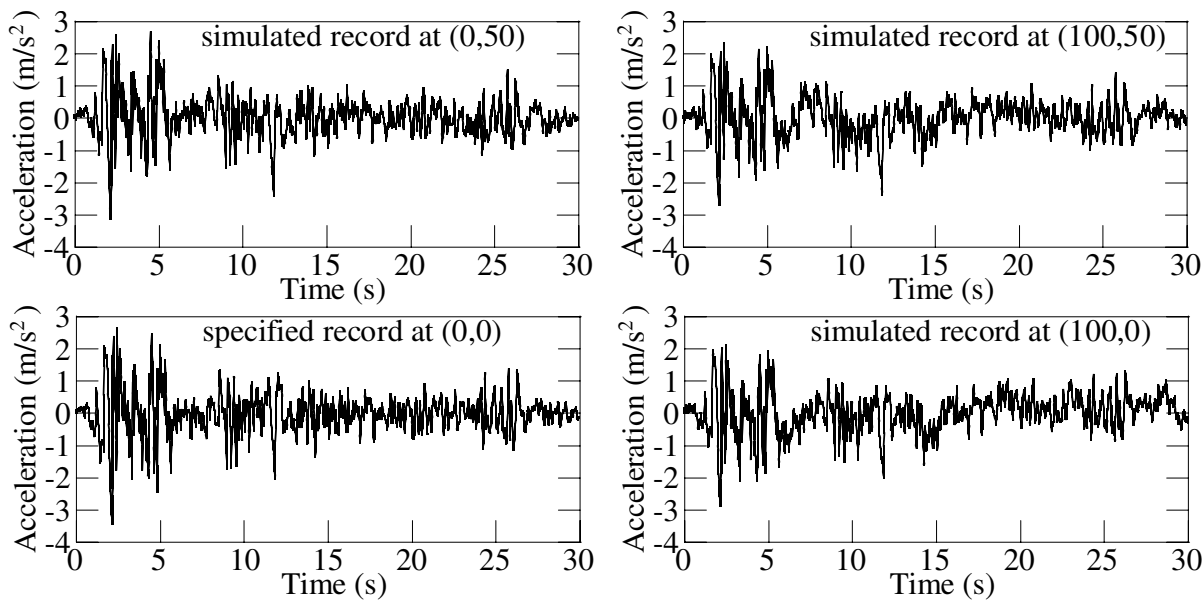

Fig. 1. Specified and simulated acceleration time histories for the El Centro earthquake

\section{Concluding Remarks}

The method of conditional stochastic simulation of ground motions using the spatiotemporal correlation function has been considered in this paper. The results of the study show that the method allows us to generate earthquake time histories with relatively low simulation errors and therefore can be suggested for practical applications.

\section{References}

1. Jankowski, R., Walukiewicz, H.: Modeling of two-dimensional random fields. Probabilistic Engineering Mechanics 12 (1997) 115-121

2. Jankowski, R., Wilde, K.: A simple method of conditional random field simulation of ground motions for long structures. Engineering Structures 22 (2000) 552-561

3. Wang, J., Carr, A., Cooke, N., Moss, P.: Effects of spatial variation of seismic inputs on bridge longitudinal response. Proc. of $13^{\text {th }}$ World Conf. on Earthquake Engineering, Vancouver, Canada (2004) paper no. 640

4. Zerva, A., Zervas, V.: Spatial variation of seismic ground motions: An overview. Applied Mechanics Reviews 55 (2002) 271-297 